\title{
Deep Palmar Arteries of Some Mammals, with Special Reference to the Palmar Metacarpal Arteries.
}

\author{
By \\ Takuro MURAKAMI, Akio KIKUTA and Takafumi NAKAI \\ Department of Anatomy, Okayama University Medical School, \\ 2-5-1 Shikata-cho, Okayama 700, Japan
}

-Received for Publication, May 28, 1987-

\begin{abstract}
Key words: forepaw, deep metacarpal arteries, palmar metacarpal arteries, proximal perforating branches, deep palmar arch

Summary: In the dog, raccoon dog, cat and rabbit, the second proximal perforating branch was fully developed forming the deep palmar arch. The catella volaris proximalis was only observed in the rabbit. The palmar metacarpal arteries arose from the deep palmar arch or catella volaris proximalis, and descended in the deep palm to finally connect with the palmar digital arteries at the end of the metacarpus. Regardless of their origin and termination, the palmar metacarpal arteries could be classified into four kinds of arteries: (1) $\mathrm{sM}$ arteries descending on the palmar surface of the interosseous muscles along the metacarpal bones, (2) sI arteries descending on the palmar surface of the interosseous muscles along the intermetacarpal spaces, (3) $\mathrm{dM}$ arteries descending deep in the interosseous muscles along the metacarpal bones, and (4) $\mathrm{dI}$ arteries descending deep in the interosseous muscles along the intermetacrapal spaces. These findings are consistant with those of the human hand and foot (Murakami, 1969, 1971).
\end{abstract}

It is well known that the deep layer of the human metacarpus is supplied by palmar metacarpal arteries. Murakami (1969) classified these metacarpal arteries in relation to the interosseous muscles and metacarpal bones: (1) superficial palmar metacarpal (sM) arteries descending on the palmar surface of the interosseous muscles along the metacarpal bones, (2) superficial palmar intermetacarpal (sI) arteries descending on the palmar surface of the interosseous muscles along the intermetacarpal spaces, (3) deep palmar metacarpal (dM) arteries descending deep in the interosseous muscles along the metacarpal bones, and (4) deep palmar intermetacarpal (dI) arteries descending deep in the interosseous muscles along the intermetacarpal spaces. Such a classification was also applicable to the human foot (Murakami, 1971). The present paper describes these four types of arteries in the forepaw of some animals.

Materials and Methods

Adult dog (Canis familialis), raccoon dog (Nyctereutes viverrinus), cat (Felis domestica) and rabbit (Oryctolagus cuniculus) were obtained from the Ritsurin Park Zoo (Takamatsu, Kagawa) upon their death. They were fixed with $10 \%$ formalin by vacular perfusion. After immersion in an ethanol bath for a year or longer, the left forepaw of these animals was dissected in 
the dissecting room of Okayama University Medical School.

\section{Results}

In the dog, raccoon dog and rabbit, the proper palmar digital arteries of the forepaw digits including the first digit arose from the palmar digital arteries which was connected to palmar branch of the ulnar artery (Fig. 1, $2,4)$. In the cat, the palmar branch of the ulnar artery was poorly developed and sup-

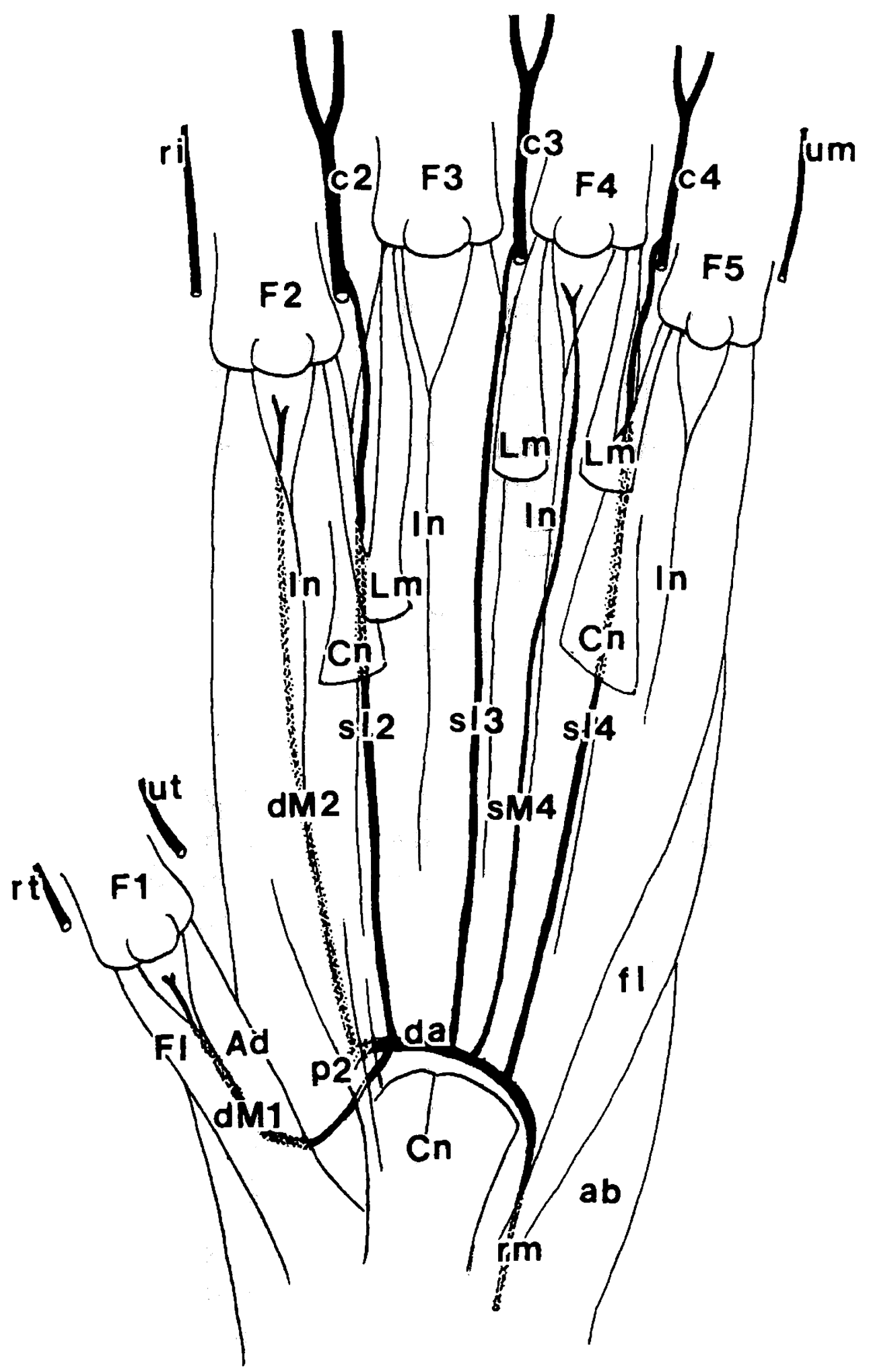

Fig. 1. A diagram of palmar view of the vascular pattern in the deep layer of the dog forepaw. Abbreviations: see Table 1. 


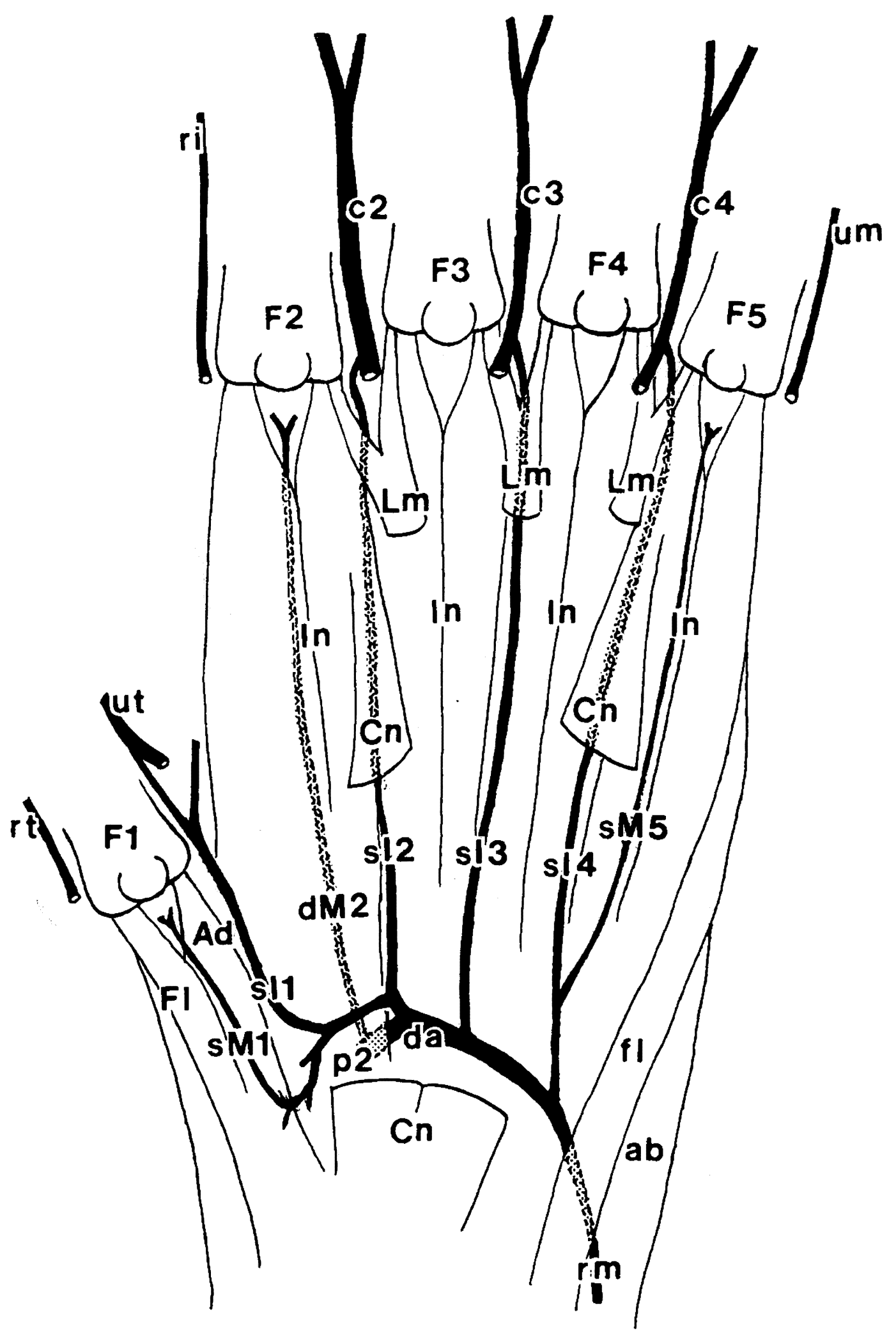

Fig. 2. A diagram of palmar view of the vascular pattern in the deep layer of the raccoon dog forepaw. Abbreviations: see Table 1.

plied only the radial proper palmar digital artery of the first digit and the ulnar proper palmar digital artery of the fifth digit; thus the remaining proper palmar digital arteries arose from the palmar metacarpal arteries in the deep palm (Fig. 3) (see below).

In all our animals, the dorsal branch of the radial artery was the origin of dorsal metacarpal arteries and proximal perforating branches at the proximal ends of the 


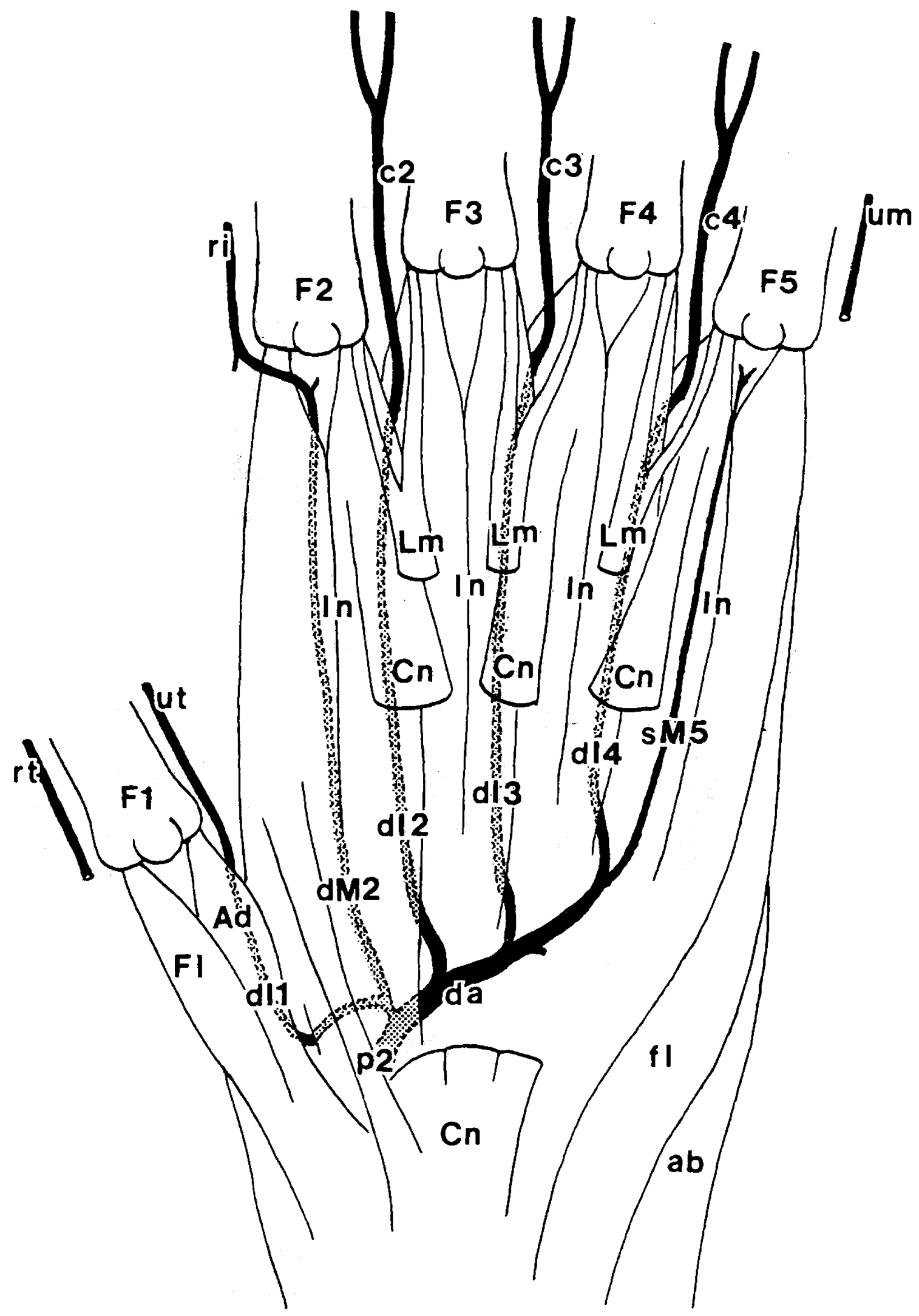

Fig. 3. A diagram of palmar view of the vascular pattern in the deep layer of the cat forepaw. Abbreviations: see Table 1. 


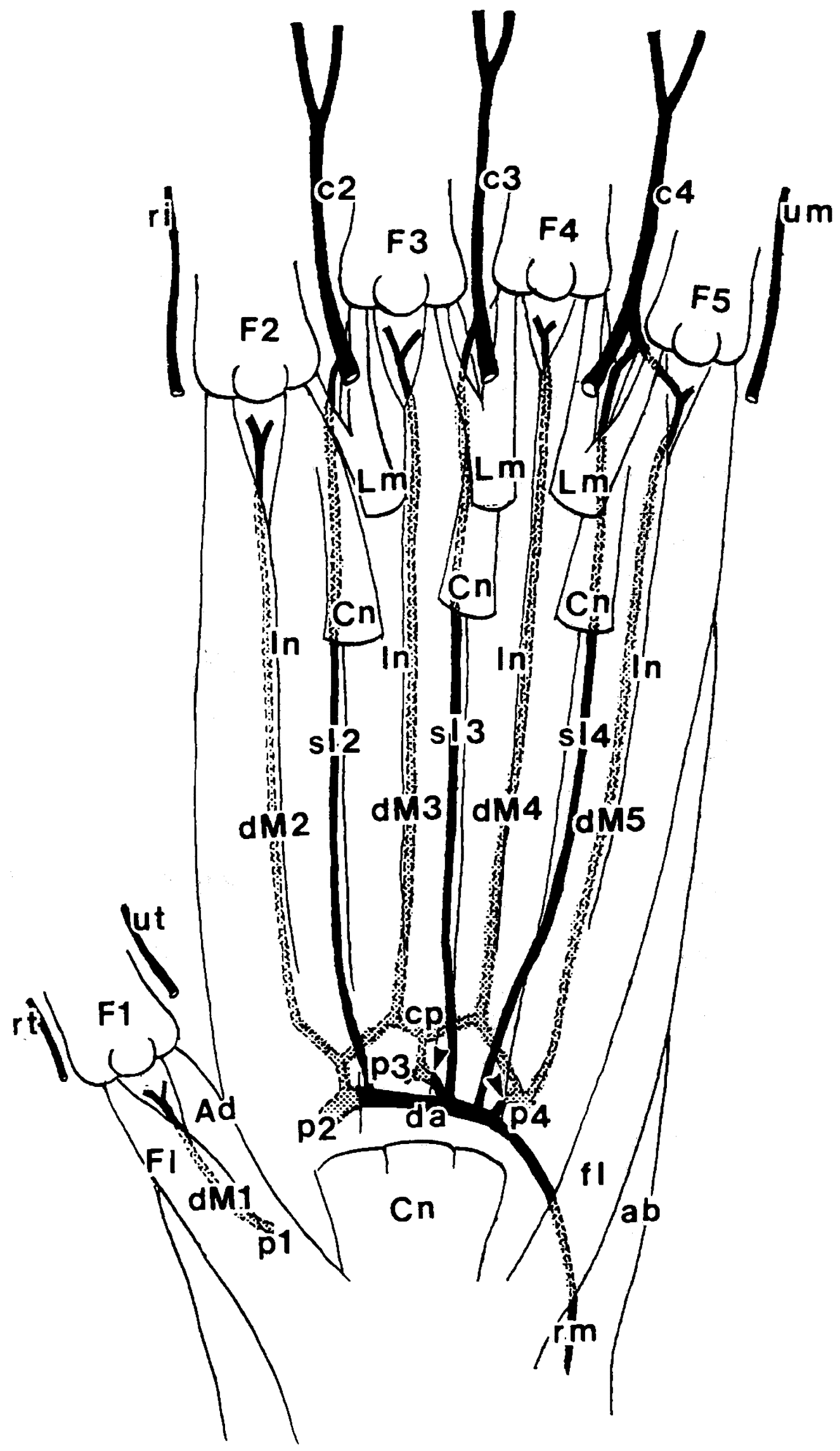

Fig. 4. A diagram of palmar view of the vascular pattern in the deep layer of the rabbit forepaw. Abbreviations: see Table 1. 
Table 1. Abbreviations in Figures $1-4$

Ad : adductor pollicis muscle

$\mathrm{Cn}$ : contrahentes muscles

F1 : flexor pollicis brevis muscle

In : interosseous muscles

Lm : lumbrical muscles

ab : abductor digiti minimi muscle

f1 : flexor digiti minimi brevis muscle

F1 - F5 : first - fifth fingers

c2 - c4 : palmar digital arteries (second - fourth common palmar digital arteries)

p1 - p4 : first - fourth proximal perforating branches

da : deep palmar arch

ri : radial proper palmar digital artery of the second finger

$\mathrm{rm}$ : deep palmar branch of the ulnar artery

rt : radial proper palmar digital artery of the first finger

um : ulnar proper palmar digital artery of the fifth finger

ut : ulnar proper palmar digital artery of the first finger

dI1 - dI4 : first - fourth deep palmar intermetacarpal arteries

dM1 - dM5 : first - fifth deep palmar metacarpal arteries

sI1 - sI4 : first - fourth superficial palmar intermetacarpal arteries

s M1 - s M5 : first - fifth superficial palmar metacarpal arteries

arrowhead : anastomoses between the deep palmar arch and catella volaris

proximalis

intermetacarpal spaces. The dorsal metacarpal arteries ran on the dorsal surface of the interosseous muscles and gave rise to the proper dorsal digital arteries which were finer than the palmar digital arteries. The proximal perforating branches penetrated the interosseous muscles at a level just distal to the base of the metacarpal bones. One of these perforating branches, the second was fully developed and passed through the second intermetacarpal space, forming the deep palmar arch on the palmar surface of the interosseous muscles with the deep palmar branch of the ulnar artery (Fig. 14). In the rabbit, the third and fourth proximal perforating branches were well developed and formed the catella volaris (palmaris) proximalis (Fig. 4) (see below). The deep palmar branch of the ulnar artery was not observed in the cat (Fig. 3).

The palmar metacarpal arteries arose from the deep palmar arch or catella volaris proximalis (Fig. 1-4). By their relationship to the interosseous muscles and metacarpal bones, the palmar metacarpal arteries except those in the pollex (see below) could be classified into: (1) the second to fifth superficial palmar metacarpal (sM2 sM5) arteries descending on the palmar surface of the interosseous muscles along the second to fifth metacarpal bones, (2) the second to fourth superficial palmar intermetacarpal (sI2 - sI4) arteries descending on the palmar surface of the interosseous muscles along the second to fourth intermetacarpal spaces, (3) the second to fifth deep palmar metacarpal (dM2 dM5) arteries descending deep in the interosseous muscles along the second to fifth metacarpal bones, and (4) the second to fourth deep palmar intermetacarpal (dI2 dI4) arteries descending deep in the interosseous muscles along the second to fourth intermetacarpal spaces.

In the pollex, the adductor pollicis muscle was small or poorly developed so that the first deep palmar intermetacarpal (dI1) and first superficial palmar intermetacarpal (sI1) artries descended respectively along the radial and ulnar margins of this muscle (Figs. 2, 3). The first deep palmar meta- 
carpal (dM1 or princeps pollicis) artery descended along the first metacarpal bone, being covered by the adductor pollicis and flexor pollicis brevis muscles (Figs. 1, 4). The first superficial palmar metacarpal (sM1) artery descended on the palmar surface of the adductor pollicis and flexor pollicis brevis muscles along the first metacarpal bone (Fig. 2).

The sM, sI, dM, and dI arteries were independent vessels, but their development varied in each case. The poorly developed $\mathrm{sM}, \mathrm{sI}, \mathrm{dM}$ and $\mathrm{dI}$ arteries terminated only in the mid-region of the metacarpus. In contrast, the well developed $\mathrm{sM}, \mathrm{sI}, \mathrm{dM}$ and $\mathrm{dI}$ arteries reached the metacarpophalangeal joints or descended over these joints to connect with the palmar digital arteries. The well developed sM, sI, dM and $\mathrm{dI}$ arteries are described.

\section{Dog (Fig. 1)}

The dorsal branch of the radial artery gave off a well dexeloped second proximal perforating branch. This branch produced the dM2 artery and gave rise to the deep palmar arch. This palmar arch received the poorly developed deep palmar branch of the ulnar artery, and gave off the dM1, sI2, sI3, sM4 and sI4 arteries. The sI2, and sI3 and sI4 arteries continued into the palmar digital arteries. The other arteries terminated near the metacarpophalangeal joints.

\section{Raccoon dog (Fig. 2)}

The dorsal branch of the radial artery gave off a well developed second proximal perforating branch. This branch yielded the $\mathrm{dM} 2$ artery and gave rise to the deep palmar arch. This palmar arch received the poorly developed deep palmar branch of the ulnar artery, and gave off the sM1, sI1, sI2, sI3, sI4 and sM5 arteries. The sI1, sI2, sI3 and
sI4 arteries continued into the palmar digital arteries. The other arteries terminated near the metacarpophalageal joints.

\section{Cat (Fig. 3)}

The dorsal branch of the radial artery gave off a well developed second proximal perforating branch. This branch produced the dI1 and dM1 arteries and gave rise to the deep palmar arch, which gave off the $\mathrm{dI} 2, \mathrm{dI} 3, \mathrm{dI} 4$ and sM5 arteries. The dI1, $\mathrm{dM} 2, \mathrm{dI} 2, \mathrm{dI} 3$ and dI4 arteries were fully developed and gave rise to the palmar digital arteries.

\section{Rabbit (Fig. 4)}

The dorsal branch of the radial artery gave off the first, second, third and fourth proximal perforating branches. The first perforating branch gave rise to the dM1 artery. The second perforating branch was well developed and gave rise to the deep palmar arch. This palmar arch received the poorly developed deep palmar branch of the ulnar artery, and gave off the sI2, sI3, and sI4 arteries. The third and fourth perforating branches anastomosed into the deep palmar arch. The third and fourth perforating branches also formed the catella volaris proximalis between the second and fifth metacarpal bones, together with a branch of the second perforating branch. This catella gave off the $\mathrm{dM} 2, \mathrm{dM} 3, \mathrm{dM} 4$ and dM5 arteries. The sI2, sI3, sI4 and dM5 arteries continued into the palmar digital arteries. The other arteries terminated near the metacarpophalangeal joints.

\section{Discussion}

This paper describes the basic vascular architecture of the palmar and dorsal layers of the forepaw and its digits of the dog, 
raccoon dog and rabbit. The structure is similar to the human hand. In these animals as in man (Adachi, 1928; Murakami, 1969), the proper palmar digital arteries (main arteries of the fingers) and the proper dorsal digital arteries (accessory arteries of the fingers) arise respectively from the palmar digital arteries and dorsal metacarpal arteries. In contrast, the cat forepaw blood vascular pattern differs somewhat. In this animal, the proper palmar digital or palmar digital arteries arise from the palmar metacarpal arteries. Such palmar digital arteries with deep origin have occasionally been reported in the human hand (Murakami, 1969). In the human foot, it has been frequently observed that the palmar metacarpal arteries with deep origin gave rise to the palmar digital arteries (Murakami, 1971).

This paper also shows that the basic blood vascular architecture of the deep layer of the forepaw of the $d o g$, raccoon dog, cat and rabbit is similar to structure in the human hand. The blood vascular system of the deep palm in these animals as in the human hand (Murakami, 1969) is composed of the superficial and deep arteries. The superficial (sM and sI) arteries descend on the palmar surface of the interosseous muscles or on the dorsal surface of the contrahentes muscles, and the deep (dM and $\mathrm{dI}$ ) arteries run deep in the interosseous muscles. The main difference is that in these animals the second proximal perforating branch is fully developed and forms the deep palmar arch, though in the human hand the first proximal perforating branch usually forms the deep palmar arch (Adachi, 1928; Murakami, 1969). In the monkey hand, the catella volaris proximalis is usually formed deep in the interosseous muscles by the first to fourth proximal perforating branches (Nishi, 1939) though it is rarely formed in the human hand (Murakami,
1969). In animals studied here, the catella volaris proximalis was observed only in the cat. In the monkey hand, the catella volaris distalis is usually formed on the palmar surface of the interosseous muscles and metacarpal bones at the distal end of the metacarpus by the distal perforating branches (Nishi, 1939). As in man (Murakami, 1969) this distal catella is poorly developed in animals studied here since the distal perforating branches are poorly developed.

This paper moreover shows that the palmar metacarpal arteries of the dog, raccoon dog, cat and rabbit as in man (Murakami, 1969) can be classified into four kinds of arteries (sM1 - sM5, sI1 - sI4, dM1 - dM5 and dI1 - dI4) in relation to the muscles and bones (see above). In the dog, raccoon dog and rabbit, the sI arteries are well developed and connect with the palmar digital arteries. In the cat, the $\mathrm{dI}$ arteries are fully developed and give rise to the palmar digital arteries. In the dog, raccoon dog, cat and rabbit, it is rather rare that the $\mathrm{sM}$ and $\mathrm{dM}$ arteries are sufficiently developed to connect with the digital arteries. However, the $\mathrm{dM}$ arteries are important in producing nutrient vessels to the shafts of the metacarpal bones. Our recent study has shown that in some monkeys such as Hylobates lar and Cynopithecus niger, $\mathrm{dM}$ arteries constantly anastomose into the catella volaris distalis. The details of these findings will be reported elsewhere. In the human hand, the main arteries in the deep palm are the sM arteries, though the $\mathrm{sI}, \mathrm{dM}$ and $\mathrm{dI}$ arteries can give rise to the palmar digital arteries (Murakami, 1969). In the human foot, the main arteries in the deep planta are also the sM arteries (Murakami, 1971).

In man, the palmar articular nerves of the second to fifth metacarpophalangeal joints arise from the deep palmar branch of the ulnar nerve and descend on the palmar surface of the interosseous muscles in close 
association with the sM arteries (Murakami, 1969). In our animals here as in man, the palmar articular nerves of the metacarpophalangeal joints arise from the deep palmar branch of the ulnar nerve and descend in close association with the $\mathrm{sM}$ arteries. In the human pollex, the palmar articular nerve of the metacarpophalangeal joint arises from the median nerve and is accompanied by the sM1 artery (Murakami, 1969). In the animals here as in man, the palmar articular nerve of the first metacarpophalangeal joint arises from the median nerve and descends in close association with the sM1 artery. This was clearly observed in the raccoon dog.

Few authors have studied the position and course of the palmar metacarpal arteries in animals. Nishi $(1939,1943)$ and Koch (1939) have studied the hands and feet of some monkeys and described two kinds of arteries; aa. metacarpicae volares and interosseae volares in the monkey hand, and aa. metatarseae plantares and interosseae plantares in the monkey foot. Judging from their description and illustrations, their aa. metacarpicae volares and metatarseae planteres correspond to our SM arteries, and their aa. interosseae volares and plantares to our dI arteries. Our recent study of the Hylobates lar, Cynopithecus niger and other monkeys has shown that the sI and $\mathrm{dM}$ arteries occur together with the $\mathrm{sM}$ and $\mathrm{dI}$ arteries. Details of these findings will be reported elsewhere. The dog palmar metacarpal arteries in the text book by Miller et al. (1964) apparently correspond to our sI arteries, judging from their positions on the palmar surface of the interosseous muscles and their course in the intermetacarpal spaces.

\section{References}

1) Adachi, B.: Arterien der Hand. Adachi, B. (ed.), Das Arteriensystem der Japaner. Band I., pp. 375-423, Maruzen Co., Kyoto (1928).

2) Koch, K.: Das Verhalten der Arterienbögen in der Vola manus und Planta pedis bei Affen. Zeitschr. Anat. Entwicklungsgesch. 110: 81-97 (1939).

3) Miller, M.E., Christensen, G.C. and Evans, H.D.: Arteries of the forepaw. Miller, M.D., Christensen, G.C. and Evans, H.D. (eds.), Anatomy of the Dog., pp. 337-341, W.B. Saunders C., Philadelphia/London (1964).

4) Murakami, T.: On the position and course of the deep palmar arteries, with special reference to the so-called palmar metacarpal arteries. Okajimas Fol. Anat. Jpn. 46: 177199 (1969).

5) Murakami, T.: On the position and course of the deep plantar arteries, with special reference to the so-called plantar metatarsal arteries. Okajimas Fol. Anat. Jpn. 48: 295322 (1971).

6) Nishi, S.: Über die Arterien der Affenhand. Ein Beitrag zur vergleichenden Angiologie. Jpn. J. Med. Sci. I. Anatomy 7: 385-393 (1939).

7) Nishi, S.: Über die Arterien des Affenfusses. Eine typologisch-anatomische Studie. Jpn. J. Med. Sci. I. Anatomy 11: 127-148 (1943). 